\title{
Digoxin Induced Sinus Bradycardia Possible Drug Interaction with Amiodarone
}

\author{
Mohamed Thayub $\mathrm{S}^{1 *}$, Neena Priyamalar $\mathrm{EM}^{2}$, Christan $\mathrm{M}^{2}$, Yadhukrishnan $\mathrm{PU}^{3}$, Daniel Sundar \\ Singh $\mathrm{S}^{2}$, Kotturathu Mammen Cherian ${ }^{4}$ \\ ${ }^{\mathrm{T}}$ Department of Clinical Pharmacy, Frontier Lifeline Hospital, Chennai, Tamil Nadu India \\ ${ }^{2}$ Department of Pharmacy Practice, C.L.Baid Metha College of Pharmacy, Chennai, Tamil Nadu India \\ ${ }^{3}$ Department of Clinical Pharmacy, Indiana Hospital and Heart Institute, Mangalore, Karnataka India \\ ${ }^{4}$ Department of Cardiothoracic Surgery, Frontier Lifeline Hospital, Chennai, Tamil Nadu India
}

*Corresponding Author

Mohamed Thayub S

\author{
Article History \\ Received: 26.01 .2020 \\ Accepted: 05.03.2020 \\ Published: 08.03 .2020
}

\begin{abstract}
Introduction: Digoxin is an inotropic drug indicated for Congestive Heart Failure (CHF) and cardiac arrhythmias. It has a narrow therapeutic index. Predisposing factors and drug-drug interactions causes digoxin toxicity at normal therapeutic doses. Hence we report a case on the adverse drug reaction of digoxin induced sinus bradycardia due to the co-administration of amiodarone with digoxin. Case report: An 83years old female patient with the known case of paroxysmal atrial fibrillation and heart failure with preserved ejection fraction. She is a known case of type 2 diabetes mellitus and hypertension. She was on treatment with digoxin $(0.25 \mathrm{mg}) 5$ days in a week along with amiodarone $200 \mathrm{mg}$ BD and other drugs. With ECG report indicating sinus bradycardia and the laboratory investigations the physician's diagnosis as an ADR caused by the drug-drug interaction resulting from the concomitant use of digoxin and amiodarone. Causality assessment was done and the drugs were withdrawn from the drug regimen. After which the patient improved symptomatically. Discussion: Sinus bradycardia is one of the early signs of digoxin toxicity. The drug interaction with amiodarone which has the potency to increase the digoxin concentration must be considered and individualization of medicines in such cases is of prime importance in order to avoid the undesired side effects or toxicity. Conclusion: Low margin of safety drug like digoxin should be prescribed with the consideration of all aspects pertaining to the individual patient. Monitoring for drug-drug interactions and also periodic Therapeutic Drug Monitoring (TDM) is beneficial.
\end{abstract}

Keywords: Digoxin Induced Drug Interaction Amiodarone.

\section{INTRODUCTION}

Digoxin is a glycosidic drug that has cardiac ionotropic property which simply means it has the potential to increase the cardiac contractility and to increase cardiac output. The mechanism of drug action is due to the reversible blockade of $\mathrm{Na}^{+} / \mathrm{K}^{+}$ATPase pump that results in the intracellular accumulation of $\mathrm{Na}^{+}$and $\mathrm{Ca}^{2+}$ as a result of which the force of contraction is increased (positive ionotropic)and by reducing the sympathetic over activity the heart rate is decreased ( negative chronotropic). It also has an extra vagal activitythat depresses the action of AV node (negative dromotropic).The main indications of digitalis are Congestive Heart Failure(CHF) and cardiac arrhythmias like atrialfibrillation (AF).Predisposing factors like advanced age, renal impairment; electrolyte imbalance, drug interaction with drugs altering digoxin metabolism lead to digoxin toxicity. Digoxin intoxication results due to the common drugs that reduce the elimination of cardiac glycosides. The (Adverse Drug Reaction) ADRs show cardiac symptoms like AV block, ventricular ectopy, ventricular fibrillation, sinus bradycardia. Anorexia, nausea, vomiting, confusion and dizziness are the majorly seen non-cardiac symptoms. Drugs like digoxin having low therapeutic index should be administered with awareness about the drug-drug interaction, not only for achieving the appropriate maintenance but also to avoid the ADRs. This is a clinical pharmacist's report on a case of Digoxin toxicity.

Copyright @ 2020: This is an open-access article distributed under the terms of the Creative Commons Attribution license which permits unrestricted use, distribution, and reproduction in any medium for non commercial use (NonCommercial, or CC-BY-NC) provided the original author and source are credited. 


\section{CASE RePOrT}

In November of 2019, a 83 years old female patient was admitted in Coronary Care Unit with complaints of recurrent vomiting since 1 week associated with giddiness. The patient was a known case of paroxysmal AF since July 2019 and a known case of Heart failure with preserved ejection fraction since 2017. She is also a known case of Type 2 Diabetes Mellitus and Systemic Hypertension. She was on Tab.Digoxin $(0.25 \mathrm{mg})$ 5days in a week along with Amiodarone 200mg twice daily since July 2019. She was also on the following drugs Tab.Atorvastatin 10mg OD, Tab. Clopidogrel 75mg OD, Tab.Pantoprazole 40mg OD, Tab.Metformin 500mg BD and Tab.Carvedilol 3.125mg BD. On General examination, patient was conscious and oriented to time, place and person. Her pulse rate was 46 beats per minute, BP was 120/80mmHg.Systemic examination was done CVS showed S1S2 +ve, RS was clear, CNS showed no abnormalities. ECG shows sinus bradycardia, ECHO shows Mild Mitral and Tricuspid valve regurgitation and severe PAH. Laboratory investigations showed serum Creatinine: $2.0 \mathrm{mg} / \mathrm{dL}$; Urea: 70mg/dL; Electrolyte $\mathrm{K}^{+}$: $4.36 \mathrm{mmol} / \mathrm{L}$,Electrolyte $\mathrm{Na}^{+}: 136.0 \mathrm{mmol} / \mathrm{L}$. Based on the above findings physician suspected it as adverse drug reaction (sinus bradycardia, A-V block).

The Drugs Digoxin and Amiodarone tend to have a major drug interaction on concomitant administration. Increased plasma digoxin levels are seen due to amiodarone co-administration. On this basis the physician diagnosis it as the digoxin induced sinus bradycardia, hence the drugs digoxin and amiodarone were withdrawn from the drug regimen. She was treated symptomatically, Laboratory values on repetition 12 hours after the initial values were serum Creatinine: 2.0mg/dL; Urea: $61 \mathrm{mg} / \mathrm{dL}$; Electrolyte $\mathrm{K}^{+}: 4.80 \mathrm{mmol} / \mathrm{L}$, Electrolyte $\mathrm{Na}^{+}: 142.0 \mathrm{mmol} / \mathrm{L}$. The patient improved symptomatically and was discharged on the $4^{\text {th }}$ day after admission. On discharge the following findings were observed Pulse rate was 54 beats per minute, BP was 110/80 mmHg. Significant improvement of the symptoms was seen after the withdrawal of drugs. The Causality assessment for the reaction was done using WHO-UMC scale which indicates possible. The reaction was reported to Pharmacovigilance programme of India via regional ADR Monitoring centre.

\section{DISCUSSION}

Digoxin is a narrow therapeutic index drug with multiple drug interactions, a slight increase of the plasma digoxin level may cause toxic reactions or may lead life threatening conditions. Drug interactions alter the pharmacokinetics and pharmacodynamics of digoxin leading to increase of digoxin levels in the body. Accumulation of digoxin happens due to the reduced elimination rate of the drug. The inotropic drug digoxin also has antiadrenergic property which causes cardiac slowing. Thus the vagal and extra vagal actions of digoxin contribute to the drug induced sinus bradycardia. Sinus bradycardia is one of the early signs of digoxin toxicity which was identified from the ECG reports of the patient. The effect of drug is the characteristic prolongation of the refractoriness of AV node and that was over expressed on exceeding the normal therapeutic index. The drug interaction with amiodarone which has the potency to increase the digoxin concentration must be considered and individualization of medicines in such cases is of prime importance in order to avoid the undesired side effects or toxicity.

\section{CONCLUSION}

Low margin of safety drug like digoxin should be prescribed with the consideration of all aspects pertaining to the individual patient. Routine therapeutic dose of digoxin exhibits toxicity due to the drug-drug interactions. Hence the management plan for a patient requiring digoxin should be planned in relation with the concomitant drug before initiation. Monitoring for drug-drug interactions and also periodic Therapeutic Drug Monitoring (TDM) in vulnerable cases is beneficial.

\section{REFERENCE}

1. Tripathi, K. (2013). Essentials of medical pharmacology. 7th ed. New Delhi: Jaypee Brothers Medical Publishers (P) Ltd, 512-520.

2. Pillay, V. (2013). Modern Medical Toxicology. 4th ed. New Delhi: Jaypee Brothers Pvt. Ltd, 318-321.

3. Nademanee, K., Kannan, R., Hendrickson, J., Ookhtens, M., Kay, I., \& Singh, B. N. (1984). Amiodarone-digoxin interaction: clinical significance, time course of development, potential pharmacokinetic mechanisms and therapeutic implications. Journal of the American College of Cardiology, 4(1), 111-116.

4. Chai, C. Y., Wang, H. H., Hoffman, B. F., \& Wang, S. C. (1967). Mechanisms of bradycardia induced by digitalis substances. American Journal of Physiology-Legacy Content, 212(1), 26-34.

5. Vyas, A., Bachani, N., Thakur, H., \& Lokhandwala, Y. (2016). Digitalis toxicity: ECG vignette. Indian heart journal, 68(Suppl 2), S223-S225.

6. MacLeod-Glover, N., Mink, M., Yarema, M., \& Chuang, R. (2016). Digoxin toxicity: Case for retiring its use in elderly patients?. Canadian Family Physician, 62(3), 223-228. 